\title{
Evidence for Coexisting Shapes through Lifetime Measurements in ${ }^{98} \mathrm{Zr}$
}

\author{
Purnima Singh, ${ }^{1}$ W. Korten, ${ }^{1}$ T. W. Hagen, ${ }^{2}$ A. Görgen, ${ }^{2}$ L. Grente,,${ }^{1}$ M.-D. Salsac,,${ }^{1}$ F. Farget, ${ }^{3}$ E. Clément, ${ }^{3}$ G. de France, ${ }^{3}$ \\ T. Braunroth, ${ }^{4}$ B. Bruyneel, ${ }^{1}$ I. Celikovic, ${ }^{3,5}$ O. Delaune, ${ }^{3}$ A. Dewald, ${ }^{4}$ A. Dijon, ${ }^{3}$ J.-P. Delaroche, ${ }^{6}$ M. Girod, ${ }^{6}$ \\ M. Hackstein, ${ }^{4}$ B. Jacquot, ${ }^{3}$ J. Libert, ${ }^{6}{ }^{\text {J. Litzinger, }}{ }^{4}$ J. Ljungvall, ${ }^{7}$ C. Louchart, ${ }^{1}$ A. Gottardo, ${ }^{8}$ C. Michelagnoli, ${ }^{8}$ \\ C. Müller-Gatermann, ${ }^{4}$ D. R. Napoli, ${ }^{8}$ T. Otsuka, ${ }^{9,10,11,12,13}$ N. Pillet, ${ }^{6}$ F. Recchia, ${ }^{14}$ W. Rother, ${ }^{4}$ E. Sahin, ${ }^{2}$ S. Siem, ${ }^{2}$ \\ B. Sulignano, ${ }^{1}$ T. Togashi, ${ }^{10}$ Y. Tsunoda,${ }^{10}$ Ch. Theisen, ${ }^{1}$ and J. J. Valiente-Dobon ${ }^{8}$ \\ ${ }^{1}$ Irfu, CEA, Université Paris-Saclay, F-91191 Gif-sur-Yvette, France \\ ${ }^{2}$ Department of Physics, University of Oslo, Oslo N-0316, Norway \\ ${ }^{3}$ Grand Accélérateur National d'Ions Lourds (GANIL), CEA/DRF-CNRS/IN2P3, \\ Boulevard Henri Becquerel, 14076 Caen, France \\ ${ }^{4}$ Institut für Kernphysik, Universität zu Köln, Köln D-50937, Germany \\ ${ }^{5}$ Vinca Institute of Nuclear Sciences, University of Belgrade, Belgrade 11000, Serbia \\ ${ }^{6}$ CEA, DAM, DIF, 91297 Arpajon, France \\ ${ }^{7}$ CSNSM, IN2P3, Orsay 91405, France \\ ${ }^{8}$ INFN, Laboratori Nazionali di Legnaro, Legnaro I-35020, Italy \\ ${ }^{9}$ Department of Physics, University of Tokyo, Hongo, Bunkyo-ku, Tokyo 113-0033, Japan \\ ${ }^{10}$ Center for Nuclear Study, University of Tokyo, Hongo, Bunkyo-ku Tokyo 113-0033, Japan \\ ${ }^{11}$ RIKEN Nishina Center, 2-1 Hirosawa, Wako, Saitama 351-0198, Japan \\ ${ }^{12}$ Instituut voor Kern- en Stralingsfysica, KU Leuven, B-3001 Leuven, Belgium \\ ${ }^{13}$ National Superconducting Cyclotron Laboratory, Michigan State University, East Lansing, Michigan 48824, USA \\ ${ }^{14}$ Dipartimento di Fisica e Astronomia "Galileo Galilei”, Università degli Studi di Padova and INFN Padova, \\ I-35131 Padova, Italy
}

(Received 11 July 2018; revised manuscript received 21 September 2018; published 9 November 2018)

The lifetimes of the first excited $2^{+}, 4^{+}$, and $6^{+}$states in ${ }^{98} \mathrm{Zr}$ were measured with the recoil-distance Doppler shift method in an experiment performed at GANIL. Excited states in ${ }^{98} \mathrm{Zr}$ were populated using the fission reaction between a $6.2 \mathrm{MeV} / \mathrm{u}{ }^{238} \mathrm{U}$ beam and a ${ }^{9} \mathrm{Be}$ target. The $\gamma$ rays were detected with the EXOGAM array in correlation with the fission fragments identified by mass and atomic number in the VAMOS++ spectrometer. Our result shows a very small $B\left(E 2 ; 2_{1}^{+} \rightarrow 0_{1}^{+}\right)$value in ${ }^{98} \mathrm{Zr}$, thereby confirming the very sudden onset of collectivity at $N=60$. The experimental results are compared to large-scale Monte Carlo shell model and beyond-mean-field calculations. The present results indicate the coexistence of two additional deformed shapes in this nucleus along with the spherical ground state.

DOI: 10.1103/PhysRevLett.121.192501

The study of various modes of excitations and the associated evolution of nuclear shapes along spin and isospin axes in atomic nuclei is one of the fundamental quests in nuclear physics. While nuclei with "magic numbers" of protons and/or neutrons have spherical ground states, as one moves away from these, the polarizing effect of added nucleons leads to deformation. Throughout the nuclear landscape, this onset of deformation is usually a gradual process; however in neutron rich nuclei around mass $A \sim 100$, the shape change is rather drastic and abrupt. The ground states of $\mathrm{Sr}$ and $\mathrm{Zr}$ isotopes with $N$ ranging from the magic number $N=50$ up to $N<60$ are weakly deformed; however, they undergo a rapid shape transition from nearly spherical to well-deformed prolate deformation as $N=60$ is approached. The sudden nature of the shape transition in $\mathrm{Sr}$ and $\mathrm{Zr}$ isotopes is evident from the abrupt changes in the two neutron separation energies [1] and the mean-square charge radii [2,3], but also from the excitation energies of $2_{1}^{+}$states and $B(E 2)$ values [4]. On the other hand, in isotopes with $Z \geq 42$, the shape change is rather gradual [1,5], also showing characteristic signatures of triaxiality. This strong dependence of the observed spectroscopic properties on the number of both protons and neutrons makes the neutron-rich $A \sim 100$ nuclei an excellent mass region for testing various theoretical models.

Many experimental and theoretical studies have already been reported on the structure of these nuclei. More specifically, for the $\mathrm{Zr}$ isotopes, the onset of deformation at $N=60$ has been described by a number of theoretical models [6-19]; however, none of the models have been able to successfully reproduce the aforementioned rapid change. Very recently, the abrupt shape changes were correctly described by large-scale Monte Carlo shell model (MCSM) calculations [20,21]. In the so-called type-II shell evolution 
scenario, the (prolate) deformed states in the isotopes with $N \geq 60$ are associated with proton excitations to the $0 g_{9 / 2}$ orbital. Driven by the central and tensor components of the effective (proton-neutron) interactions, these excitations result in a lowering and subsequent filling of the neutron $0 g_{7 / 2}$ and $0 h_{11 / 2}$ orbitals [21]. Therefore, both protons and neutrons act coherently to induce the deformation. These prolate deformed states, based on multiparticle-multihole excitations, are expected to coexist with the spherical ground state in $\mathrm{Zr}$ isotopes with $N<60$. The crossing of these two distinct coexisting quantum configurations at $N=60$ manifests as the abrupt change in the structure of the ground state and has been interpreted as a quantum phase transition (QPT) from a spherical to a deformed phase [21].

The appearance of low-lying $\mathrm{O}_{2}^{+}$states in $\mathrm{Sr}$ and $\mathrm{Zr}$ isotopes with $N<60$ supports the shape coexistence scenario in these isotopes as an explanation of the rapid shape evolution. However, in order to have a complete picture of these phenomena, it is essential to have precise information on the deformation as inferred from the electromagnetic transition strengths for nuclei in the region of the shape transition. Experimental programs pursued in the past to determine these parameters in the $A \sim 100$ region nuclei have been able to furnish useful insight into the phenomena [22-25]. For example, the coexistence of highly deformed prolate and spherical configurations has already been established in ${ }^{96,98} \mathrm{Sr}[22,23]$ and ${ }^{94,96} \mathrm{Zr}[24,25]$.

Shape coexistence has also been suggested in ${ }^{98} \mathrm{Zr}$ $[26,27]$, a key nucleus for understanding the QPT phenomenon in the $\mathrm{Zr}$ isotopes. Several attempts have been made in the past to determine the electromagnetic transition rates in ${ }^{98} \mathrm{Zr}$. In previous work by Bettermann et al. [28], using the $\beta \gamma \gamma$ fast-timing method, a lifetime of $\tau\left(4^{+}\right)=$ $29 \pm 9$ ps was obtained for the $4^{+}$state in ${ }^{98} \mathrm{Zr}$, while only upper limits could be obtained for the $2^{+}$state $\left[\tau\left(2_{1}^{+}\right) \leq 15 \mathrm{ps}\right]$ and the $6^{+}$state $\left[\tau\left(6_{1}^{+}\right) \leq 14 \mathrm{ps}\right]$. More recently, in the work by Ansari et al. [29], the so-far most precise upper limit for the lifetime of the $2_{1}^{+}$state in ${ }^{98} \mathrm{Zr}$ was obtained as $\tau\left(2_{1}^{+}\right) \leq 6 \mathrm{ps}$, and an upper limit of $15 \mathrm{ps}$ was reported for the $4_{1}^{+}$state. These limits for the $2^{+}$state did not allow us to prove whether the onset of collectivity at $N=60$, i.e., in ${ }^{100} \mathrm{Zr}$, is as rapid as expected from the drop in excitation energy, and as predicted by the MCSM, or whether the $B(E 2)$ value already increases in ${ }^{98} \mathrm{Zr}$. Moreover, in the absence of precise information on $B(E 2)$ values, one cannot draw any definite conclusion about the properties of the coexisting structures.

The present Letter reports the results of lifetime measurement of the first excited $2^{+}, 4^{+}$, and $6^{+}$states in ${ }^{98} \mathrm{Zr}$ using the recoil-distance Doppler shift method and confirms for the first time shape coexistence in this nucleus. In addition, our result shows a very small $B\left(E 2 ; 2_{1}^{+} \rightarrow 0_{1}^{+}\right)$ value in ${ }^{98} \mathrm{Zr}$, thereby confirming the very sudden onset of collectivity at $N=60$.

The excited states in ${ }^{98} \mathrm{Zr}$ were produced in fission reactions, where a ${ }^{238} \mathrm{U}$ beam with an energy of 6.2 MeV/u delivered by the GANIL facility impinged on a $2.3 \mathrm{mg} / \mathrm{cm}^{2}$-thick ${ }^{9} \mathrm{Be}$ target. The VAMOS++ [30,31] spectrometer positioned at $20^{\circ}$ with respect to the beam axis was used to detect and identify the reaction products by mass, charge, and atomic number on an event-by-event basis. Gamma rays emitted from the reaction products were detected using the EXOGAM array [32] consisting of ten segmented Clover detectors arranged in two rings (three detectors at $135^{\circ}$ and seven detectors at $90^{\circ}$ with respect to the spectrometer axis). Correlation between the prompt $\gamma$ rays detected at the target position and the detection of an ion in the focal plane of VAMOS++ triggered the event building, thus allowing the selection of prompt $\gamma$ rays from isotopically identified fission fragments.

A compact plunger device was used for the lifetime measurements using the recoil-distance Doppler shift (RDDS) technique [33]. The recoiling ${ }^{98} \mathrm{Zr}$ nuclei had an average velocity of $37.3 \mu \mathrm{m} / \mathrm{ps}$ and were slowed down in a $4.9 \mathrm{mg} / \mathrm{cm}^{2}$-thick $\mathrm{Mg}$ degrader foil placed behind the target. Data were collected for seven distances ranging from 37 to $1554 \mu \mathrm{m}$ with average running times of approximately 24 hours per distance.

The energy of the $\gamma$ rays was Doppler-corrected event by event using the measured velocity and direction of the fission fragments as measured after the degrader foil in VAMOS++. Figure 1 shows the Doppler-corrected $\gamma$-ray spectra of ${ }^{98} \mathrm{Zr}$ observed with the EXOGAM detectors located at $135^{\circ}$ for different target-degrader distances. The $\gamma$ rays that were emitted after passing through the degrader appear at the correct transition energy in the Dopplercorrected spectra, whereas those emitted between the target and degrader are shifted to lower energies when observed at backward angles. The lifetimes of individual states were determined from the relative intensities of the two components as a function of target-degrader distance using the differential decay curve method (DDCM) analysis for singles plunger data [34] (also see the Supplemental Material [35]). All observed feeders have been taken into account as per their experimentally observed intensities (see the Supplemental Material [35]). The unobserved feeding contribution was subtracted following the procedure explained in Ref. [34]. We have also explored independently the influence on lifetimes due to relativistic effects, possible deorientation effects and solid angle effects; however, contributions due to these effects were found negligible as compared to the experimental uncertainties. Further details on the experiment and the analysis techniques can be found in Refs. [36,37].

As the most important result, the lifetime of the $2_{1}^{+}$state was determined for the first time as $\tau=3.8 \pm 0.8 \mathrm{ps}$. In addition, we were also able to determine the lifetimes for 


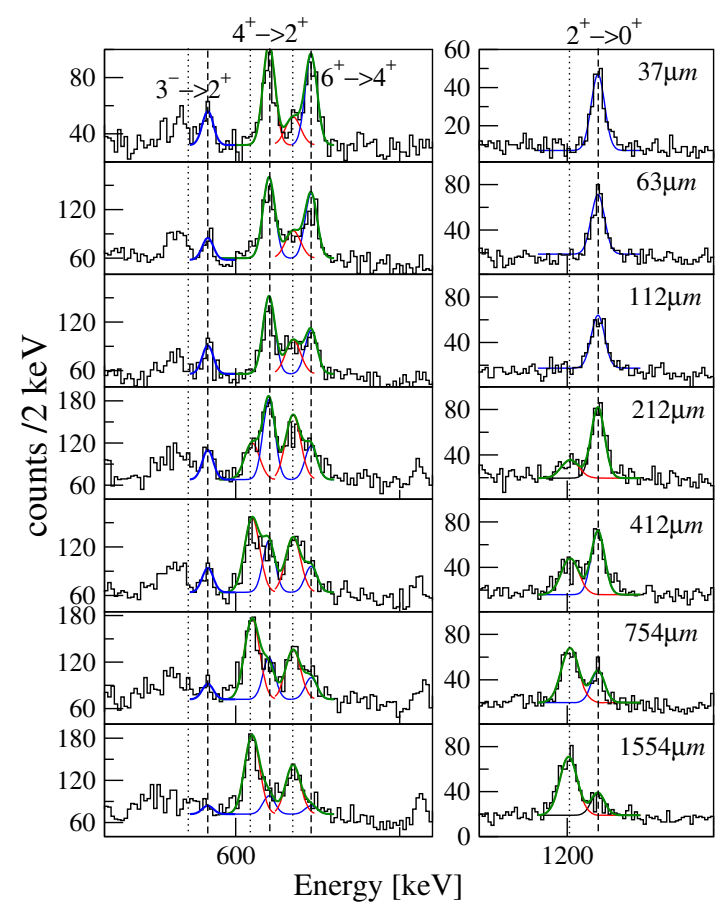

FIG. 1. Gamma-ray spectra showing the $2^{+} \rightarrow 0^{+}, 4^{+} \rightarrow 2^{+}$, $6^{+} \rightarrow 4^{+}$, and $3^{-} \rightarrow 2^{+}$transitions in ${ }^{98} \mathrm{Zr}$, observed in the detectors at $135^{\circ}$ for seven target-degrader distances. The spectra are Doppler-corrected using the velocity measured in VAMOS++ after the degrader. The dotted and dashed lines indicate the positions of the shifted and unshifted components, respectively. Also displayed are the Gaussian fits to the spectra (green), and the individual fits to the shifted (red) and unshifted (blue) components.

the $4_{1}^{+}$and the $6_{1}^{+}$states as $7.5 \pm 1.5 \mathrm{ps}$ and $2.6 \pm 0.9 \mathrm{ps}$, respectively. The $B(E 2)$ values from the present work are listed in Table I. Our new lifetime measurement yields a $B\left(E 2 ; 2_{1}^{+} \rightarrow 0_{1}^{+}\right)$value of $2.9(0.6)$ W.u., thereby confirming the rapid onset of deformation in $\mathrm{Zr}$ isotopes only at $N=60$.

The onset of deformation in the $\mathrm{Zr}$ isotopes has been described by a number of theoretical models. Information from $B(E 2)$ values on the rapid structural changes in neutron-rich $\mathrm{Sr}$ and $\mathrm{Zr}$ isotopes is available from calculations

TABLE I. Summary of results for the lifetime measurements in ${ }^{98} \mathrm{Zr}$.

\begin{tabular}{lccc}
\hline \hline$I_{i}^{\pi} \rightarrow I_{f}^{\pi}$ & $\tau_{\exp }(\mathrm{ps})$ & $\tau_{\text {lit }}(\mathrm{ps})$ & $B(E 2, \downarrow)_{\exp }(\mathrm{W} . \mathrm{u})$. \\
\hline $2_{1}^{+} \rightarrow 0_{1}^{+}$ & $3.8(0.8)$ & $\leq 6[29]$ & $2.9(0.6)^{\mathrm{a}}(0.2)^{\mathrm{b}}$ \\
$2_{1}^{+} \rightarrow 0_{2}^{+}$ & & & $28.3(6.0)^{\mathrm{a}}(2.4)^{\mathrm{b}}$ \\
$4_{1}^{+} \rightarrow 2_{1}^{+}$ & $7.5(1.5)$ & $\leq 15[29]$ & $43.3(8.7)^{\mathrm{a}}(10.8)^{\mathrm{b}}$ \\
$4_{1}^{+} \rightarrow 2_{2}^{+}$ & & & $67.5(13.5)^{\mathrm{a}}(16.9)^{\mathrm{b}}$ \\
$6_{1}^{+} \rightarrow 4_{1}^{+}$ & $2.6(0.9)$ & & $103.0(35.7)^{\mathrm{a}}$ \\
\hline \hline
\end{tabular}

The errors in the $B(E 2)$ values from the measured lifetimes ${ }^{\mathrm{a}}$ and from the branching ratios ${ }^{b}$ taken from Ref. [38] are mentioned separately. solving a five-dimensional collective Hamiltonian $(5 \mathrm{DCH})$ with parameters determined within the relativistic mean-field approach (PC-PK1 force) [39], the nonrelativistic HartreeFock approach using the SLy4 [39], and the Gogny-D1S [8] force. All these calculations suggest a picture of sphericaloblate-prolate shape transition in the neutron-rich $\mathrm{Sr}$ and $\mathrm{Zr}$ isotopes.

Figure 2 compares the $B\left(E 2 ; 2_{1}^{+} \rightarrow 0_{1}^{+}\right)$values from these models with the experimental data. It can be clearly noted that the models based on the mean-field approach predict a gradual increase in collectivity already starting at $N \sim 54$. This is also the case for the interacting boson model (IBM) calculations using self-consistent mean-field approximation based on the Gogny-D1M energy density functional [18]. However, certain differences can be noted depending on the underlying effective force. The PC-PK1 and Gogny D1S forces predict a very gradual change in most of the collective properties between $N=54$ and $N=60$. Calculations using the SLy4 force, on the other hand, predict a more pronounced change in collectivity at $N=60$ [39]. None of them is, however, capable of reproducing the experimental data. This could in part be related to the fact that the particle-number projection in these methods is only valid on average. Simply speaking, there might still be components in the theoretical wave functions from the neighboring isotopes with $N \pm 2$, which would smoothen out the evolution with neutron number. It is also worth noting that the conventional large-scale shell model (SM) calculations [12] show a rather accurate reproduction of experimental data up to $N=58$, but they are constrained by valence space limitation to $N<60$. Finally, the dramatic increase of $B\left(E 2 ; 2_{1}^{+} \rightarrow 0_{1}^{+}\right)$values between $N=58$ and 60 is very well reproduced by recent state-of-the-art MCSM calculations [21].

Using the most recent branching ratios determined by Urban et al. in the $\beta$ decay of ${ }^{98} \mathrm{Y}$ [38], we were also able to determine the $B(E 2)$ values for the decay to the nonyrast

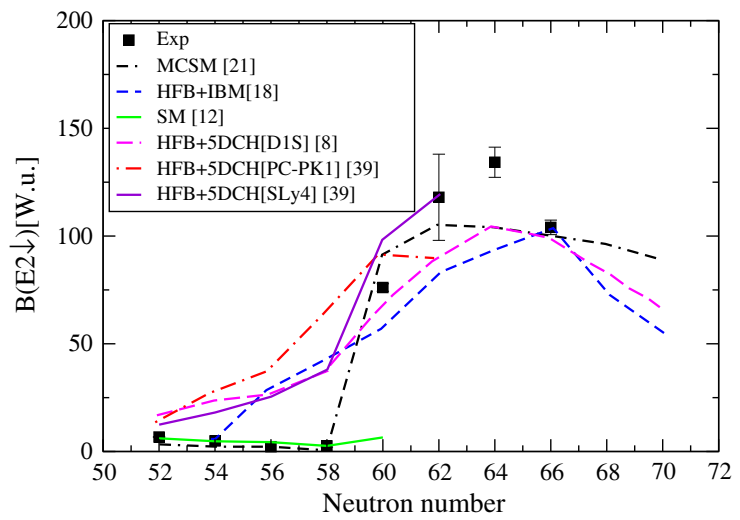

FIG. 2. Comparison of calculated $B\left(E 2 ; 2_{1}^{+} \rightarrow 0_{1}^{+}\right)$values with experimental data for the $\mathrm{Zr}$ isotopes. Experimental data points are from Refs. [24,29,40-42]. Theoretical values are taken from Refs. [8,12,18,21,39]. 


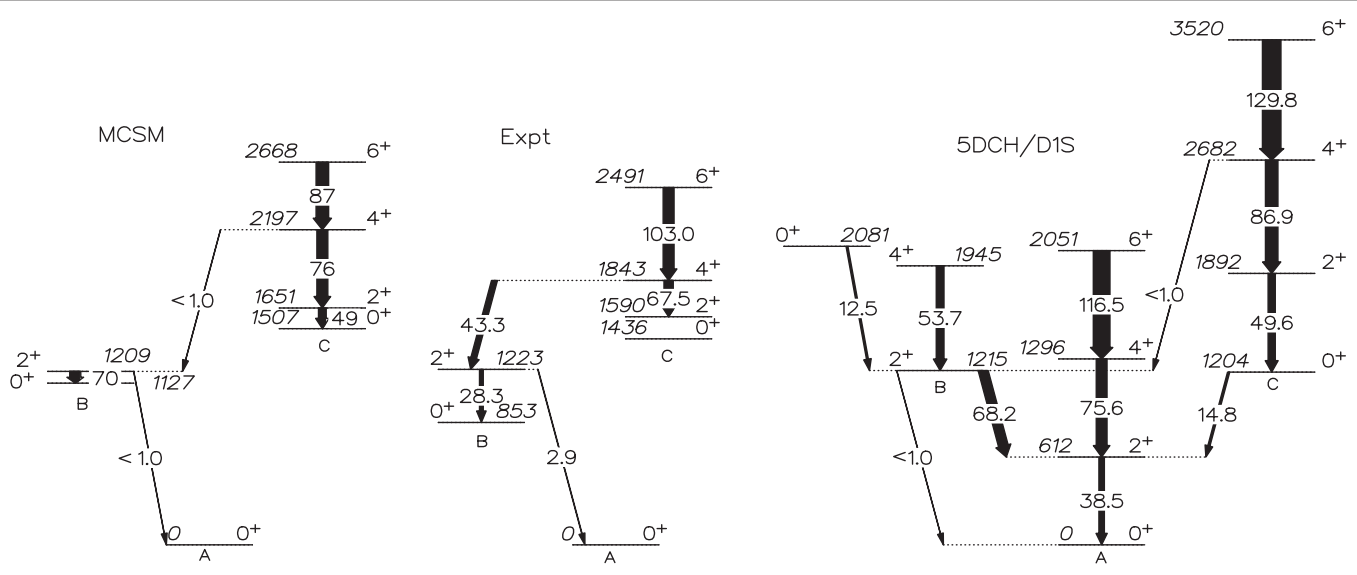

FIG. 3. Comparison between the experimental and theoretical level schemes for ${ }^{98} \mathrm{Zr}$. The excitation energy and spin are mentioned against each state. Labels and widths of the arrows represent calculated [8,21] and measured $B(E 2)$ values in W.u.

states, which give important new information. The experimental excitation energies of the states and the transition strengths between them are compared with the calculations in Fig. 3. A collective value of $B\left(E 2 ; 2_{1}^{+} \rightarrow 0_{2}^{+}\right)=$ 28.3(6.4) W.u. suggests a common moderately deformed structure for the $0_{2}^{+}$and $2_{1}^{+}$states. Assuming a rigid, axial symmetric deformed shape yields a quadrupole deformation parameter $\beta_{2} \approx 0.21$. These results are in general agreement with the results of MCSM calculations, which predict an enhanced $B\left(E 2 ; 2_{1}^{+} \rightarrow 0_{2}^{+}\right)$and very small $B\left(E 2 ; 2_{1}^{+} \rightarrow 0_{1}^{+}\right)$transition strengths. However, while the calculations suggest a gradual increase in deformation of the $0_{2}^{+}$states between ${ }^{94} \mathrm{Zr}$ and ${ }^{98} \mathrm{Zr}$ [21], our present results indicate a deformation which is smaller than that observed in ${ }^{96} \mathrm{Zr}$ [25].

As shown in Fig. 3, the $B(E 2)$ values in ${ }^{98} \mathrm{Zr}$ indicate the coexistence of three different structures at low spin: A nearly spherical $0_{1}^{+}$ground state, a moderately deformed excited $\mathrm{O}_{2}^{+}$state as discussed above, and a well-deformed (bandlike) structure possibly based on the $0_{3}^{+}$state. The existence of the latter is supported by the rather large $B(E 2)$ values within this band, as shown in Fig. 3. A significant mixing of this well-deformed structure with the moderately deformed configuration, based on the $0_{2}^{+}$state, is indicated by the large $B\left(E 2 ; 4_{1}^{+} \rightarrow 2_{1}^{+}\right)$value. A strong mixing between the two $2^{+}$states was already suggested in Ref. [26] based on their similar feeding and decay patterns. Finally, the large electric monopole transition strength observed between the $0_{3}^{+}$and $0_{2}^{+}$states also supports the mixing of two coexisting shapes [27].

The proposed triple shape coexistence scenario in ${ }^{98} \mathrm{Zr}$ is also supported by MCSM calculations, which predict the coexistence of three shapes in this nucleus: a spherical $0_{1}^{+}$ state, a prolate deformed $\mathrm{O}_{2}^{+}$state, and a triaxial $\mathrm{O}_{3}^{+}$state. The underlying mechanism for stabilizing such coexisting structures has been discussed in terms of type-II shell evolution [21]. As shown in Fig. 4, the calculations clearly indicate that all the relevant states above $0_{1}^{+}$involve on the average three proton excitations from the $1 p 0 f$ orbitals to the $0 g_{9 / 2}$ orbital and five neutron excitations from the $1 d_{5 / 2} 2 s_{1 / 2}$ orbitals to the $0 g_{7 / 2}, 1 d_{3 / 2}$, and $0 h_{11 / 2}$ orbitals. The structures of the $0_{2}^{+}$and $2_{1}^{+}$states are similar to each other. The $0_{3}^{+}$state and all the other states built on top of it also have similar configurations. The $B(E 2)$ values within the band predicted from MCSM calculations are very well in agreement with the experiment for the structure based on $0_{3}^{+}$. However, it is to be noted that the MCSM
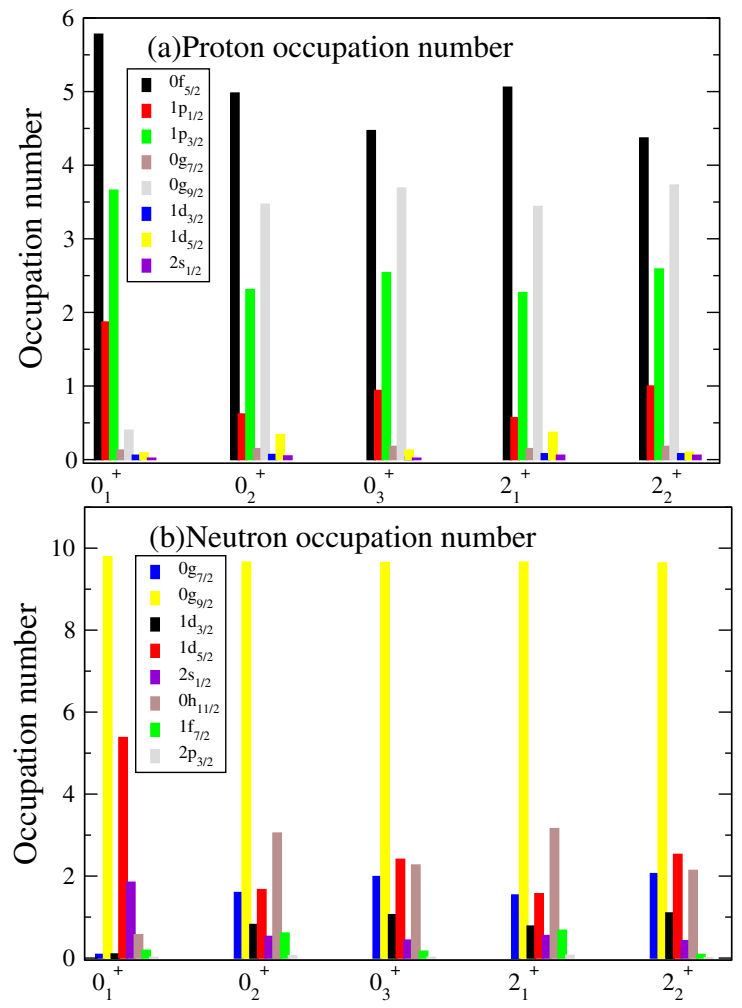

FIG. 4. Occupation numbers of (a) protons and (b) neutrons for the states in ${ }^{98} \mathrm{Zr}$. 
calculations do not reproduce the strong mixing of the two $2^{+}$states (see Fig. 3). The observed discrepancies suggest a need for further refinement of the shell-model Hamiltonian used in the present MCSM calculations.

The five-dimensional beyond-mean-field calculations based on the Gogny force (5DCH/D1S) overestimate in general the deformation in ${ }^{98} \mathrm{Zr}$. These calculations suggest a $K=0$ yrast band structure based on the $0_{1}^{+}$state, which gets stretched with increasing angular momentum, i.e., with mean shape coordinates $\beta$ and $\gamma$ changing from 0.23 to 0.37 and $27^{\circ}$ to $16^{\circ}$, respectively. The calculations also predict a second well-deformed prolate band including the levels $\mathrm{O}_{2}^{+}$, $2_{3}^{+}, 4_{3}^{+}, 6_{3}^{+}, 8_{3}^{+}$. This band is predicted to be more rigid against triaxiality $\left(\gamma \sim 18^{\circ}\right)$; furthermore, the mean axial deformation $\beta$ is changing from 0.34 to 0.42 . The transition strengths within the excited prolate band are at low spin rather close to the experimental values of the band built on the $0_{3}^{+}$state, but they increase more strongly within the band. The deformed structures are therefore reasonably well reproduced by this model; however it fails to reproduce the spherical ground state of ${ }^{98} \mathrm{Zr}$, a feature which was also observed in other mass regions and also explains the smooth onset of collectivity at $N=60$ observed for all mean field calculations (see Fig. 2).

To summarize, using the recoil-distance Doppler shift method on isotopically identified fission fragments, we have measured the lifetimes in the neutron-rich isotope ${ }^{98} \mathrm{Zr}$, which is located just below the predicted quantum phase transition at $N=60$. The result shows a very small $B\left(E 2 ; 2_{1}^{+} \rightarrow 0_{1}^{+}\right)$value in ${ }^{98} \mathrm{Zr}$, confirming the very sudden onset of collectivity at $N=60$. This effect is well described by the MCSM calculations and interpreted as quantum phase transition. Beyond-mean-field calculations are in general not able to reproduce this effect, which could be related to the treatment of the particle-number projection. The results from the present measurement confirm the coexistence of three distinct shapes in this nucleus for the first time. A comparison of the measured $B(E 2)$ values with the state-of-the-art MCSM calculations indicate a spherical-prolate-triaxial shape coexistence in ${ }^{98} \mathrm{Zr}$.

The authors thank the technical teams at Grand Accélérateur National d'Ions Lourds for their support during the experiment and the authors of Ref. [43] for preparing and optimising the VAMOS++ and EXOGAM spectrometers. We also thank A. Navin for helpful discussions. This work has received funding from the European Union's Horizon 2020 research and innovation program under Marie Skłodowska-Curie Grant Agreement No. 702590. It was also supported by the European Community FP7 Integrated Infrastructure Initiative, Contract ENSAR No. 262010, by the Research Council of Norway under Project Grants No. 213442 and No. 263030, and by Deutsche Forschungsge-meinschaft (DFG) Project No. DE 1516/3-1. The MCSM calculations were performed on a K computer at
RIKEN AICS (hp150224, hp160211). This work was supported in part by the HPCI Strategic Program (The origin of matter and the universe) and "Priority Issue on postK computer" (Elucidation of the Fundamental Laws and Evolution of the Universe) from MEXT and Joint Institute for Computational Fundamental Science.

[1] U. Hager et al., Phys. Rev. Lett. 96, 042504 (2006).

[2] F. Buchinger et al., Phys. Rev. C 41, 2883 (1990).

[3] P. Campbell et al., Phys. Rev. Lett. 89, 082501 (2002).

[4] National Nuclear Data Center, Brookhaven National Laboratory, http://www.nndc.bnl.gov/.

[5] F. C. Charlwood et al., Phys. Lett. B 674, 23 (2009).

[6] J. Skalski, P.-H. Heenen, and P. Bonche, Nucl. Phys. A559, 221 (1993).

[7] R. Rodríguez-Guzmán, P. Sarriguren, L. M. Robledo, and S. Perez-Martin, Phys. Lett. B 691, 202 (2010).

[8] J.-P. Delaroche, M. Girod, J. Libert, H. Goutte, S. Hilaire, S. Péru, N. Pillet, and G. F. Bertsch, Phys. Rev. C 81, 014303 (2010); (private communication).

[9] P. G. Reinhard, D. J. Dean, W. Nazarewicz, J. Dobaczewski, J. A. Maruhn, and M. R. Strayer, Phys. Rev. C 60, 014316 (1999).

[10] J. Skalski, S. Mizutory, and W. Nazarewicz, Nucl. Phys. A617, 282 (1997).

[11] A. Holt, T. Engeland, M. Hjorth-Jensen, and E. Osnes, Phys. Rev. C 61, 064318 (2000).

[12] K. Sieja, F. Nowacki, K. Langanke, and G. MartínezPinedo, Phys. Rev. C 79, 064310 (2009).

[13] Y.-X. Liu, Y. Sun, X.-H. Zhou, Y.-H. Zhang, S.-Y. Yu, Y.-C. Yang, and H. Jin, Nucl. Phys. A858, 11 (2011).

[14] A. Petrovici, Phys. Rev. C 85, 034337 (2012).

[15] C. Özen and D. J. Dean, Phys. Rev. C 73, 014302 (2006).

[16] J. E. García-Ramos, K. Heyde, R. Fossion, V. Hellemans, and S. De Baerdemacker, Eur. Phys. J. A 26, 221 (2005).

[17] M. Böyükata, P. Van Isacker, and İ. Uluer, J. Phys. G 37, 105102 (2010).

[18] K. Nomura, R. Rodríguez-Guzmán, and L. M. Robledo, Phys. Rev. C 94, 044314 (2016).

[19] J. Xiang, Z. P. Li, Z. X. Li, J. M. Yao, and J. Meng, Nucl. Phys. A873, 1 (2012).

[20] T. Otsuka and Y. Tsunoda, J. Phys. G 43, 024009 (2016).

[21] T. Togashi, Y. Tsunoda, T. Otsuka, and N. Shimizu, Phys. Rev. Lett. 117, 172502 (2016).

[22] E. Clément et al., Phys. Rev. Lett. 116, 022701 (2016).

[23] E. Clément et al., Phys. Rev. C 94, 054326 (2016).

[24] A. Chakraborty, E. E. Peters, B. P. Crider et al., Phys. Rev. Lett. 110, 022504 (2013).

[25] C. Kremer et al., Phys. Rev. Lett. 117, 172503 (2016).

[26] C. Y. Wu, H. Hua, D. Cline, A. B. Hayes, R. Teng, R. M. Clark, P. Fallon, A. Görgen, A. O. Macchiavelli, and K. Vetter, Phys. Rev. C 70, 064312 (2004).

[27] K. Heyde and J. L. Wood, Rev. Mod. Phys. 83, 1467 (2011). 
[28] L. Bettermann, J.-M. Régis, T. Materna, J. Jolie, U. Köster, K. Moschner, and D. Radeck, Phys. Rev. C 82, 044310 (2010).

[29] S. Ansari et al., Phys. Rev. C 96, 054323 (2017).

[30] S. Pullanhiotan, M. Rejmund, A. Navin, W. Mittig, and S. Bhattacharyya, Nucl. Instrum. Methods Phys. Res., Sect. A 593, 343 (2008).

[31] M. Rejmund et al., Nucl. Instrum. Methods Phys. Res., Sect. A 646, 184 (2011).

[32] J. Simpson, F. Azaiez, G. de France, J. Fouan, J. Gerl, R. Julin, W. Korten, P. Nolan, B. Nyak, G. Sletten, and P. Walker (EXOGAM Collaboration), Acta Phys. Hung. 11, 159 (2000).

[33] A. Dewald, O. Möller, and P. Petkov, Prog. Part. Nucl. Phys. 67, 786 (2012).

[34] A. Dewald, S. Harissopulos, and P. von Brentano, Z. Phys. A 334, 163 (1989).

[35] See Supplemental Material at http://link.aps.org/ supplemental/10.1103/PhysRevLett.121.192501 for the decay curves and partial level scheme of ${ }^{98} \mathrm{Zr}$ which were used in the present analysis.

[36] T. W. Hagen et al., Phys. Rev. C 95, 034302 (2017).

[37] T. W. Hagen et al., Eur. Phys. J. A 54, 50 (2018).

[38] W. Urban et al., Phys. Rev. C 96, 044333 (2017).

[39] H. Mei, J. Xiang, J. M. Yao, Z. P. Li, and J. Meng, Phys. Rev. C 85, 034321 (2012).

[40] S. Raman, C. W. Nestor, Jr., and P. Tikkanen, At. Data Nucl. Data Tables 78, 1 (2001).

[41] G. Kumbartzki, N. Benczer-Koller, J. Holden et al., Phys. Lett. B 562, 193 (2003).

[42] F. Browne, A. M. Bruce, T. Sumikama et al., Phys. Lett. B 750, 448 (2015).

[43] A. Navin, M. Rejmund, C. Schmitt, S. Bhattacharyya, G. Lhersonneau, P. V. Isacker, M. Caamano, E. Clément, O. Delaune, F. Farget, G. de France, and B. Jacquot, Phys. Lett. B 728, 136 (2014). 